\title{
PIM1 kinase as a promise of targeted therapy in prostate cancer stem cells (Review)
}

\author{
YINGQIU XIE* and SAMAT BAYAKHMETOV* \\ Department of Biology, Nazarbayev University School of Science and Technology, Astana 010000, Republic of Kazakhstan
}

Received July 2, 2015; Accepted October 20, 2015

DOI: $10.3892 / \operatorname{mco} .2015 .673$

\begin{abstract}
Since the last decade, the PIM family serine/threonine kinases have become a focus in cancer research. Numerous clinical data supports that overexpression of PIM1 is associated with tumor formation in various tissues. However, little is known regarding the function of PIM1 in cancer stem cells. In cancer cells, PIM1 has essential roles in the regulation of the cell cycle, cell proliferation, cell survival and multiple drug resistance. In stem cells, PIM1 kinase exhibits a significant function in stem cell proliferation, self-renewal and expansion. Thus, PIM1 shows a great promise in cancer therapy by targeting stem cells. Furthermore, it is imperative to investigate Pim-1 targeting in cancer stem cells by applicable inhibitors for improving future outcomes. The present review investigated the potential of PIM1 as a therapy target in prostate cancer stem cells.
\end{abstract}

\section{Contents}

1. Introduction

2. Canonical PIM1 signaling

3. PIM1 promotes multiple drug resistance: A phenotype of cancer stem cells

4. PIM1 functions in prostate cancer: AR downregulation and Myc activation

5. PIM1 functions in stem cells

6. PIM1 functions in prostate stem/cancer stem cells

7. Targeting PIM1 for inhibition of cancer stem cells

8. Future directions

Correspondence to: Dr Yingqiu Xie, Department of Biology, Nazarbayev University School of Science and Technology, 53 Kabanbay Batyr Avenue, Astana 010000, Republic of Kazakhstan

E-mail: yingqiu.xie@nu.edu.kz

${ }^{*}$ Contributed equally

Key words: PIM1, cancer stem cell, prostate cancer

\section{Introduction}

Currently, cancer is the leading cause among death-associated diseases. In particular, prostate cancer $(\mathrm{PCa})$ is the second most common cause of cancer-related fatalities among men in the USA. Androgen deprivation is currently the standard therapy for aggressive prostate cancer through inhibition of androgen receptor (AR) activity; however, castration-resistant prostate cancer (CRPC), an advanced and metastatic type of cancer, may develop (1). CRPC is one of the lethal forms of malignancy relapse in prostate cancer patient mortality. Recurrence of cancer is caused by a subset of the cancer cell population, known as cancer stem cells; however, the molecular mechanisms underlying how the signaling pathways may promote cancer relapse through cancer stem cells are largely unknown. The cancer stem cells occupy only a small percentage of the subpopulation of cancer cells (2); however, they possess all the major features of stem cells, including self-renewal, unlimited growth and the ability to differentiate to any type of tissue (3). The difference between stem cells and cancer stem cells is that cancer stem cells are resistant to chemotherapeutic drugs and are involved in cancer cell signaling (4). Therefore, understanding the molecular pathways of cancer development within these cells may allow us to explore the specific agents that target these cells (5). Thus far, some of the agents have been identified as anticancer drugs and are widely used in the targeted therapies (6). In particular, small molecular kinase inhibitors have been investigated and have entered the clinical trials for targeting cancer stem cells (7). These compounds are able to target kinases, which are identified as overexpressed in numerous solid tumors and hematopoietic malignancies. The PIM1 kinase inhibitors recently have shown promise with regards to anticancer properties (8). In the present review, the role of PIM1 in cancer stem cells is evaluated with regards to the novel avenues that may be explored for future prostate cancer treatment.

\section{Canonical PIM1 signaling}

The PIM serine/threonine kinases have been widely studied from the early 1990s. The name of PIM originates from the proviral insertion site in murine lymphomas (9), known as Moloney Murine Leukemia Virus (10). PIM1 is one of the three members in the PIM family (11). Even though these 
three share $>60$ of homolog amino acids, only PIM1 has a significant impact on tumor formation (9). In particular, PIM1 alone can induce cancer progression in the prostate as a proto-oncogene (12); however, in cooperation with Myc, it may enhance the prostate tumorigenesis in vivo (13).

The human PIM1 protein is translated from the PIMI gene, which is $5-\mathrm{kb}$ with a transcript of 2,984 base pairs (14). This gene encodes for two isoforms of the protein, which are 33 (PIM1S) and 44 kDa (PIM1L) with single mRNA (15), differential starting codon AUG and atypical CUG, respectively (11). Our previous study reported that PIM1S mainly localizes to the cytosol and nucleus; however, PIM1L predominately localizes at the plasma membrane (16). Therefore, distinct cellular localization may affect their function. In tissues, PIM1 is usually expressed in hematopoietic tissues, such as the thymus, spleen, bone marrow and fetal liver, and also in the hippocampus, oral epithelia and prostate (17).

The PIM1 kinase is extremely unique due to its constitutive activation. Therefore, activation of PIM1 largely depends on the elevation of its mRNA or proteins levels, which are found in numerous cancers (18). Expression of PIM1 is mainly induced by cytokines (17). In addition, nuclear factor- $\kappa \mathrm{B}$, Jak-signal transducer and activator of transcription (STAT), ETS-related gene and hypoxia-inducible factor-1 $\alpha$ are the most major pathways that induce PIM1 upregulation (18-20). The downstream targets of PIM1 signaling are typically regulated by direct phosphorylation by PIM1. Thus far, 30 substrates interact with and are phosphorylated by PIM1. Through phosphorylation of target proteins, PIM1 has essential roles in the regulation of the cell cycle, cell proliferation, anti-apoptosis, multiple drug resistance, chromatin remodeling, protein translation, energy metabolism and stress response $(9,21,22)$. The following focuses on PIM1 functions in association with stem/cancer stem cells.

\section{PIM1 promotes multiple drug resistance: A phenotype of cancer stem cells}

In several studies it was shown that PIM1 has a crucial role in resistance to chemotherapy drugs. According to our previous study, the PIM1L isoform, which has an additional N-terminal proline-rich region with the same kinase domain as PIM1S, promotes prostate cancer cell resistance to chemotherapy drugs, such as mitoxantrone and docetaxel, which are approved by Food and Drug Administration for prostate cancer treatment (16). Mechanically, the proline-rich domain of PIM1L directly interacts with the SH3 domain of Etk, and thereby competes with p53 in binding to Etk due to anti-apoptosis induced by chemotherapeutic drugs (16), promoting their cell surface expression. Membrane localization is known to be crucial for efflux activity of drug transporters at the plasma membrane. Our previous studies reported that PIM1 promotes membrane translocation of the drug transporters breast cancer resistance protein (BCRP)/adenosine triphosphate-binding cassette sub-family $\mathrm{G}$ member 2 (ABCG2) and P-glycoprotein (Pgp)/ABCB1 $(23,24)$. Thus, PIM1 elevation enhances the drug resistance activity of prostate cancer cells. As BCRP is a putative stem cell marker (25), while drug resistance is the main characteristic of cancer stem cells, PIM1 most likely regulates cancer stem cells.

\section{PIM1 functions in prostate cancer: AR downregulation and Myc activation}

Studies on PIM1 function in cancer stem cells remain limited. However, there are numerous studies regarding the function of PIM1 in different stem cells and solid cancers, particularly prostate cancer, bladder cancer (26) and urothelial carcinomas (27).

The functions of PIM1 in cancer cells have been investigated in numerous types of cancer. In prostate cancer, PIM1 is considered a major biomarker. PIM1S (28) and PIM1L (16) can be used as a prognostic marker in advanced prostate cancer. Using high-density tissue microarrays consisting of $\sim 700$ human prostate cancer specimens, PIM1S overexpression was identified in $51 \%$ of samples (28). PIM1S overexpression also predicates the prostate-specific antigen recurrence (28). Our previous study reported a similar result of PIM1L in prostate cancer specimens (16).

$\mathrm{AR}$ is the master regulator of prostate cancer. Our previous study identified that AR phosphorylation is essential for prostate cancer progression and CRPC development (29). Furthermore, another previous study reported that PIM1 directly interacts with and phosphorylates AR. In detail, AR stability is regulated through phosphorylation by the two isoforms of PIM1 (PIM1S and PIM1L). PIM1S mediates AR Ser-213 phosphorylation and degrades AR by ubiquitin E3 ligase mouse double minute 2 homolog; however, PIM1L mediates Thr-850 phosphorylation to stabilize AR (30). In addition, AR was thought to inhibit stem/progenitor cells self-renewal (31) and AR loss has been found to enhance the cancer stem cell properties through interleukin 6 (IL-6)/STAT3 signaling (32). As PIM1 is a component of the IL-6-STAT3 pathway, PIM1 most likely mediates the crosstalk of AR with STAT3 in cancer stem cells.

In human prostate cancer specimens, PIM1 and Myc have been found to be co-upregulated (28). Myc is one of the four transcription factors that induce pluripotent stem cells (iPS) through nuclear reprogramming. In a prostate cancer xenograft model, single PIM1 overexpression only induced low-grade prostatic intraepithelial neoplasia (PIN) lesions, and Myc alone only induced adenocarcinoma or carcinoma with neuroendocrine differentiation (33). Using tissue recombination assays, PIM1 and Myc co-expression induced high-grade PIN and invasive adenocarcinoma. Mechanically, PIM1 increased Myc transcriptional activity (34) through stabilizing Myc by phosphorylation (35). However, whether PIM1 and Myc cooperate to promote cancer progression through Myc-mediated reprogramming remains to be elucidated.

\section{PIM1 functions in stem cells}

Increasing evidence has shown the functions of PIM1 in stem cells, including embryonic, mesenchymal, hematopoietic, cardiac and prostate stem cells. In mice, Pim1 expression is induced by STAT3 and leukemia inhibitory factor (LIF) in embryonic stem (ES) cells at the transcriptional level. The elevated Pim1 thereby promotes the self-renewal ability of ES cells (35). As Myc (36) and Kruppel-like factor 4 (Klf4) (37), two of the reprogramming factors for iPS, can also be induced by LIF, Pim1 may cooperate with the cell reprogramming in ES cells upon LIF stimulation. Our previous study showed that IL-6 can upregulate PIM1S and PIM1L in prostate cancer 
cells (16). Notably, in ES cells fused to human fibroblasts, IL-6 treatment upregulates Myc. Cooperating with the other three reprograming factors, octamer-binding transcription factor 4 (Oct4), Klf4 and Sox2, IL-6 also upregulates Pim1 to increase the iPS frequency (38). This evidence indicates the role of PIM1 in iPS through IL-6/STAT3 and the Myc pathway. In addition, in rat mesenchymal stem cells, sheer stress can induce PIM1 transcription and thereby PIM1 enhances the proliferation of mesenchymal stem cells (39). In an ovine model, PIM1 sustains the mesenchymal stem cells proliferation and survival upon hypoxia or serum deprivation (40).

A previous study in Japan using Pim1 transgenic mice showed that the number of $\mathrm{Lin}^{-} \mathrm{Sca}^{+} \mathrm{c}-\mathrm{Kit}^{+}$hematopoietic stem/progenitor cells was increased compared to normal mice (41). The study also reported that knockout mice of Pim1, but not Pim2 or Pim3, showed the impaired long-term hematopoietic repopulating capacity in secondary and competitive transplantations (41). In another example, FMS-like tyrosine kinase 3 mutation of internal tandem duplication (FLT3-ITD) results in constitutive activation of FLT3 independent for proliferation and survival of leukemic cells. FLT3-ITD enhances human cluster of differentiation (CD)34(+) haematopoietic stem/progenitor cells self-renewal ability and transformation in vitro (42) and in mouse models (43). Our previous study showed that PIM1 directly interacts and phosphorylates FLT3-ITD at Serine 951 thereby stabilizing FLT3-ITD (44). These data suggest that PIM1 may enforce FLT3-ITD-mediated transformation of stem cells. Another example of PIM1 function in stem cells was identified by experiments with human cardiac stem/progenitor cells (hCPCs). These experiments used heart biopsy samples with C-kit-positive hCPCs and identified that PIM1-transduced cells showed a decreased senescence phenotype with increased telomere length and telomerase activity (45). PIM1 overexpressed hCPCs also showed increased proliferation and cell survival (46).

\section{PIM1 functions in prostate stem/cancer stem cells}

PIM1 is essential to prostate stem and cancer stem cells as well. As mentioned above, PIM1 phosphorylates and enhances the activity of BCRP/ABCG2, a putative stem cell marker. Our previous study further identified the essential role of PIM1 in prostate tumor initiation through cancer stem-like cells. To the best of our knowledge, the study was the first to report the co-upregulation of PIM1 and BCRP in multiple drug resistance prostate cancer cell lines that we established (23). The drug resistant cell line that highly expressed BCRP showed an increased side population (46). The side population is a subset of cells enriched with BCRP, which can efflux a specific Hoechst 33342 dye and were originally identified to present the stem cells in mouse bone marrow (47). In mouse prostate, single cells expressing putative stem cell markers, $\mathrm{Lin}^{-} \mathrm{Sca}^{+} \mathrm{CD} 133^{+} \mathrm{CD} 44^{+}$ $\mathrm{CD}_{117^{+}}$or Epcam ${ }^{+} \mathrm{CD} 44^{+} \mathrm{CD} 49 \mathrm{f}^{\text {hi }}$, can generate prostate-like tissues followed by implantation under the renal capsule of host mice of compromised immunity (48). By contrast, expressing BCRP is sufficient for side population cells isolated from human prostate cancer specimens to engraft the prostatic duct regeneration using similar tissue recombination with urogenital sinus mesenchyme (rUGM) assays (48). However, without recombination with rUGM, expressing BCRP is not sufficient to support

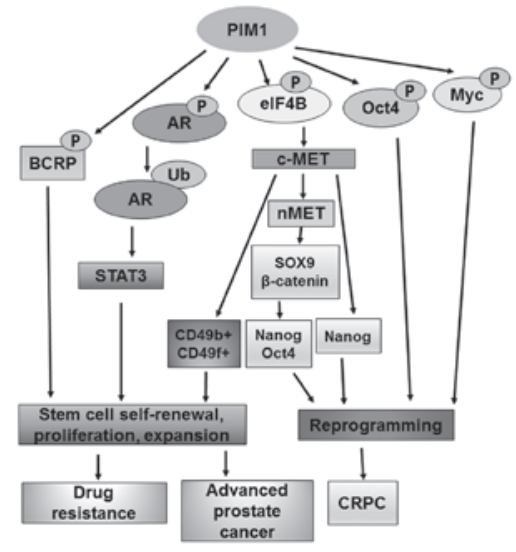

Figure 1. PIM1 signaling pathways are associated with stem/cancer stem cells in the prostate. The potential linkages between PIM1 phosphorylation of substrates and regulation of stem/cancer stem cells or reprogramming are proposed.

the engrafted duct growth. This is consistent with another study, which reported that the side population of $\mathrm{BCRP}^{+}$compared to $\mathrm{BCRP}^{-}$cells isolated from xenograft prostate tumor alone do not show a distinct ability for tumorigenesis, and even express stemness genes, such as Notch-1 and $\beta$-catenin (49). These data suggest that additional signaling is required for BCRP-mediated tumor initiation, while PIM1 signaling is most likely one of them. Further investigations on PIM1 function in stemness should be performed using cancer stem cells expressing multiple cancer stem cell markers.

In addition to BCRP, our previous study also found that Oct4 is required for the tumor initiation driven by as low as 100 cells in a xenograft prostate cancer model (46). Another study reported the co-upregulation of PIM1 and OCT4 in the drug-resistant prostate cancer (50). The phosphorylation of Oct 4 by PIM1 has been reproducibly confirmed by nano-flow liquid chromatography mass spectrometry and mutagenesis analysis by another lab using ES cells (51). However, in ES cells the phosphorylation of Oct4 decreases iPS efficiency (51). Therefore, the differential effect of PIM1 in ES cells and cancer stem cells may suggest the essential role of the crosstalk of PIM1 with oncogenic signaling networks in the distinction of stem cell and cancer stem cell.

c-MET kinase has been shown to promote glioblastoma cell reprogramming through Nanog to maintain the stem-like character (52). In prostate cancer cells, c-MET induces stem-like properties by upregulation of stem cell markers of CD49b, CD49f and CD44 (53). Recently, it has been shown that PIM1 can upregulate c-MET expression through enhanced translation via Serine 406 phosphorylation of eukaryotic initiation factor 4B by PIM1 (54). This study indicates that PIM1 may be an upstream factor of the c-MET signaling pathway that regulates stemness. Furthermore, our recent study showed that a nuclear form of c-MET, known as nMET, can induce prostate cancer cell reprogramming and maintain stemness through SOX9, $\beta$-catenin, Oct4 and Nanog (55). nMET subsequently reactivates AR through $\beta$-catenin. Most notably, nMET was upregulated in $75 \%$ of CRPC patient specimens (55). As nMET is dependent on c-MET expression, PIM1 may indirectly regulate nMET-mediated prostate cancer cell reprogramming. In conclusion, PIM1 may activate multiple signaling of cancer 
stem cells through phosphorylation, such as BCRP and Myc (Fig. 1). Therefore, PIM1 is most likely a promising target in cancer stem cell-targeted therapy.

\section{Targeting PIM1 for inhibition of cancer stem cells}

As PIM1 has great potential to induce cancer stem cell properties to accelerate prostate cancer progression, targeting PIM1 in cancer stem cells using selective inhibitors may provide a new avenue for treatment of aggressive prostate cancer. The early generation of PIM1 inhibitor, SGI-1776, has entered the phase I clinical trial of castration-resistant prostate cancer patients (27). SGI-1776 inhibits not only prostate cancer cell growth, but also PIM1-mediated Taxane resistance, through inhibition of drug transporter Pgp (56). Our previous study later identified the mechanism that SGI-1776 inhibits PIM1-mediated protection of Pgp from proteasomal degradation and decreases Pgp cell surface expression, thereby increasing Pgp overexpressing cell sensitization to the chemotherapy drugs (24). ABC transporter-mediated drug resistance is common in cancer stem cells, so targeting PIM1-mediated reversal of drug resistance may be through cancer stem cell inhibition. However, the severe side effect of cardiotoxicity resulted in the termination of the trials (27).

The new generation of the PIM1 inhibitor, TP-3654, was screened as the lead compound targeting PIM1 kinase (27). Using the PC3 cell-derived xenograft model, oral gavage at a dose of $200 \mathrm{mg} / \mathrm{kg}$ of TP-3654 significantly decreased the growth of tumors (27). However, further signaling analysis of cancer stem cells affected by TP-3654 is lacking. AZD1208 is another newly screened highly selective inhibitor that decreases Myc/PIM1 graft tumor growth by $54.3 \pm 39 \%(\mathrm{P}<0.001)(57)$. In particular, combined with radiation treatment and upregulation of p53, AZD1208 showed more efficiency in the inhibition of prostate cancer cell growth (57). Whether AZD1208 can inhibit PIM1-mediated cancer stem cell proliferation, expansion and cell reprogramming by targeting Myc remains to be elucidated.

\section{Future directions}

There is no empirical evidence for the PIM1 direct function in prostate cancer stem cells and PIM1 inhibitor efficiency in direct targeting of cancer stem cells. However, taking into consideration that targeted therapies using PIM1 inhibitors are already in process, further investigations of the PIM1 function in prostate cancer stem cells in vivo should be performed using a genetically engineered mouse model of prostate cancer and should examine the pharmacological efficiency of applicable PIM1 inhibitors in prostate cancer stem cell-induced tumor initiation. As opposed to assessing the solid and already differentiated prostate tumors, the prostate cancer stem cells may prove viable for investigation. One important piece of evidence for this proposal is that other kinase pathways in cancer stem cells have been well studied, and therefore, targeting PIM1 may inhibit prostate tumor initiation induced by cancer stem cells.

In conclusion, the effective targeted therapy for prostate cancer remains questionable. PIM1 inhibitors are showing great promise; however, the vast majority of known treatments exhibit numerous adverse side effects. Additionally, experiments for these treatments are only performed in malignant tissues, whereas cancer stem cell-targeted therapies with PIM1 expression are not performed. Therefore, conducting further research in this area may aid in generating an efficient treatment against prostate cancer.

\section{Acknowledgements}

The present study was supported in part by the seed grant from Nazarbayev University (Y.X.).

\section{References}

1. Huggins C and Hodges CV: Studies on prostatic cancer. I. The effect of castration, of estrogen and androgen injection on serum phosphatases in metastatic carcinoma of the prostate. CA Cancer J Clin 22: 232-240, 1972.

2. Lawson DA and Witte ON: Stem cells in prostate cancer initiation and progression. J Clin Invest 117: 2044-2050, 2007.

3. Bonnet D and Dick JE: Human acute myeloid leukemia is organized as a hierarchy that originates from a primitive hematopoietic cell. Nat Med 3: 730-737, 1997.

4. Hanahan D and Weinberg RA: The hallmarks of cancer. Cell 100: 57-70, 2000.

5. Hanahan D and Weinberg RA: Hallmarks of cancer: The next generation. Cell 144: 646-674, 2011.

6. Stegmeier F, Warmuth M, Sellers WR and Dorsch M: Targeted cancer therapies in the twenty-first century: Lessons from imatinib. Clin Pharmacol Ther 87: 543-552, 2010.

7. Nawijn MC, Alendar A and Berns A: For better or for worse: The role of Pim-1 oncogenes in tumorigenesis. Nat Rev Cancer 11: 23-34, 2011.

8. Arunesh GM, Shanthi E, Krishna MH, Sooriya Kumar J and Viswanadhan VN: Small molecule inhibitors of Pim-1 kinase: July 2009 to February 2013 patent update. Expert Opin Ther Pat 24: 5-17, 2014.

9. Brault L, Gasser C, Bracher F, Huber K, Knapp S and Schwaller J: PIM serine/threonine kinases in the pathogenesis and therapy of hematologic malignancies and solid cancers. Haematologica 95: 1004-1015, 2010.

10. Hoover D, Friedmann M, Reeves R and Magnuson NS: Recombinant human pim-1 protein exhibits serine/threonine kinase activity. J Biol Chem 266: 14018-14023, 1991.

11. Saris CJ, Domen J and Berns A: The pim-1 oncogene encodes two related protein-serine/threonine kinases by alternative initiation at AUG and CUG. EMBO J 10: 655-664, 1991.

12. Kim J, Roh M and Abdulkadir SA: Pim1 promotes human prostate cancer cell tumorigenicity and c-MYC transcriptional activity. BMC Cancer 10: 248, 2010.

13. Wang J, Kim J, Roh M, Franco OE, Hayward SW, Wills ML and Abdulkadir SA: Pim1 kinase synergizes with c-MYC to induce advanced prostate carcinoma. Oncogene 29: 2477-2487, 2010.

14. Bullock AN, Debreczeni J, Amos AL, Knapp S and Turk BE: Structure and substrate specificity of the Pim-1 kinase. J Biol Chem 280: 41675-41682, 2005.

15. Jacobs MD, Black J, Futer O, Swenson L, Hare B, Fleming M and Saxena K: Pim-1 ligand bound structures reveal the mechanism of serine/threonine kinase inhibition by LY294002. J Biol Chem 280: 13728-13734, 2005.

16. Xie Y,Xu K, Dai B, GuoZ, Jiang T, Chen H and Qiu Y: The 44 kDa Pim-1 kinase directly interacts with tyrosine kinase Etk/BMX and protects human prostate cancer cells from apoptosis induced by chemotherapeutic drugs. Oncogene 25: 70-78, 2006.

17. Eichmann A, Yuan L, Bréant C, Alitalo K and Koskinen PJ: Developmental expression of pim kinases suggests functions also outside of the hematopoietic system. Oncogene 19: 1215-1224, 2000.

18. Bachmann $\mathrm{M}$ and Möröy T: The serine/threonine kinase Pim-1. Int J Biochem Cell Biol 37: 726-730, 2005.

19. Merkel AL, Meggers E and Ocker M: PIM1 kinase as a target for cancer therapy. Expert Opin Investig Drugs 21: 425-436, 2012.

20. Magistroni V, Mologni L, Sanselicio S, Reid JF, Redaelli S, Piazza R, Viltadi M, Bovo G, Strada G, Grasso M, et al: ERG deregulation induces PIM1 over-expression and aneuploidy in prostate epithelial cells. PLoS One 6: e28162, 2011. 
21. Wang Z, Bhattacharya N, Mixter PF, Wei W, Sedivy J and Magnuson NS: Phosphorylation of the cell cycle inhibitor p21Cip1/WAF1 by Pim-1 kinase. Biochim Biophys Acta 1593, $45-55,2002$.

22. Aho TL, Sandholm J, Peltola KJ, Mankonen HP, Lilly M and Koskinen J: Pim-1 kinase promotes inactivation of the pro-apoptotic Bad protein by phosphorylating it on the Ser112 gatekeeper site. FEBS Letters 571: 43-49, 2004

23. Xie Y, Xu K, Linn DE, Yang X, Guo Z, Shimelis H, Nakanishi T, Ross DD, Chen H, Fazli L, et al: The 44-kDa Pim-1 kinase phosphorylates BCRP/ABCG2 and thereby promotes its multimerization and drug-resistant activity in human prostate cancer cells. J Biol Chem 283: 3349-3356, 2008.

24. Xie Y, Burcu M, Linn DE, Qiu Y and Baer MR: Pim-1 kinase protects P-glycoprotein from degradation and enables its glycosylation and cell surface expression. Mol Pharmacol 78: 310-318, 2010.

25. Krishnamurthy P, Ross DD, Nakanishi T, Bailey-Dell K, Zhou S, Mercer KE, Sarkadi B, Sorrentino BP and Schuetz JD: The stem cell marker Bcrp/ABCG2 enhances hypoxic cell survival through interactions with heme. J Biol Chem 279: 24218-24225, 2004.

26. Guo S, Mao X, Chen J, Huang B, Jin C, Xu Z and Qiu S: Overexpression of Pim-1 in bladder cancer. J Exp Clin Cancer Res 29: 161, 2010

27. Foulks JM, Carpenter KJ, Luo B, Xu Y, Senina A, Nix R, Chan A, Clifford A, Wilkes M, Vollmer D, et al: A small-molecule inhibitor of PIM kinases as a potential treatment for urothelia carcinomas. Neoplasia 16: 403-412, 2014.

28. Dhanasekaran SM, Barrette TR, Ghosh D, Shah R, Varambally S, Kurachi K, Pienta KJ, Rubin MA and Chinnaiyan AM: Delineation of prognostic biomarkers in prostate cancer. Nature 412: 822-826, 2001.

29. Guo Z, Dai B, Jiang T, Xu K, Xie Y, Kim O, Nesheiwat I, Kong X, Melamed J, Handratta VD, et al: Regulation of androgen receptor activity by tyrosine phosphorylation. Cancer Cell 10: 309-319, 2006.

30. Linn DE, Yang X, Xie Y, Alfano A, Deshmukh D, Wang X, Shimelis $\mathrm{H}$, Chen $\mathrm{H}, \mathrm{Li} \mathrm{W}, \mathrm{Xu} \mathrm{K}$, et al: Differential regulation of androgen receptor by PIM-1 kinases via phosphorylation-dependent recruitment of distinct ubiquitin E3 ligases. J Biol Chem 287: 22959-22968, 2012.

31. Huang CK, Luo J, Lee SO and Chang C: Concise review: Androgen receptor differential roles in stem/progenitor cells including prostate, embryonic, stromal and hematopoietic lineages. Stem Cells 32: 2299-2308, 2014

32. Schroeder A, Herrmann A, Cherryholmes G, Kowolik C, Buettner R, Pal S, Yu H, Müller-Newen G and Jove R: Loss of androgen receptor expression promotes a stem-like cell phenotype in prostate cancer through STAT3 signaling. Cancer Res 74: 1227-1237, 2014.

33. Wang J, Kim J, Roh M, Franco OE, Hayward SW, Wills ML and Abdulkadir SA: Pim1 kinase synergizes with c-MYC to induce advanced prostate carcinoma. Oncogene 29: 2477-2487, 2010.

34. Zhang Y, Wang Z, Li X and Magnuson NS: Pim kinase-dependent inhibition of c-Myc degradation. Oncogene 27: 4809-4819, 2008

35. Aksoy I, Sakabedoyan C, Bourillot PY, Malashicheva AB, Mancip J, Knoblauch K, Afanassieff $M$ and Savatier P: Self-renewal of murine embryonic stem cells is supported by the serine/threonine kinases Pim-1 and Pim-3. Stem Cells 25: 2996-3004, 2007.

36. Cartwright P, McLean C, Sheppard A, Rivett D, Jones K and Dalton S: LIF/STAT3 controls ES cell self-renewal and pluripotency by a Myc-dependent mechanism. Development 132: 885-896, 2005

37. Li Y, McClintick J, Zhong L, Edenberg HJ, Yoder MC and Chan RJ: Murine embryonic stem cell differentiation is promoted by SOCS-3 and inhibited by the zinc finger transcription factor Klf4. Blood 105: 635-637, 2005.

38. Brady JJ, Li M, Suthram S, Jiang H, Wong WH and Blau HM: Early role for IL-6 signalling during generation of induced pluripotent stem cells revealed by heterokaryon RNA-Seq. Nat Cell Biol 15: 1244-1252, 2013

39. Tu ML, Wang HQ, Sun XD, Chen LJ, Peng XC, Yuan YH, Li RM, Ruan XZ, Li DS, Xu YJ and Ke ZJ: Pim-1 is up-regulated by shear stress and is involved in shear stress-induced proliferation of rat mesenchymal stem cells. Life Sci 88: 233-238, 2011.

40. Zhao Y, Hu J, Buckingham B, Pittenger MF, Wu ZJ and Griffith BP: Pim-1 kinase cooperates with serum signals supporting mesenchymal stem cell propagation. Cells Tissues Organs 199: 140-149, 2014
41. An N, Lin YW, Mahajan S, Kellner JN, Wang Y, Li Z, Kraft AS and Kang Y: Pim1 serine/threonine kinase regulates the number and functions of murine hematopoietic stem cells. Stem Cells 31: 1202-1212, 2013

42. Li L, Piloto O, Kim KT, Ye Z, Nguyen HB, Yu X, Levis M, Cheng L and Small D: FLT3/ITD expression increases expansion, survival and entry into cell cycle of human haematopoietic stem/progenitor cells. Nat Cell Biol 137: 64-75, 2007.

43. Chu SH, Heiser D, Li L, Kaplan I, Collector M, Huso D, Sharkis SJ, Civin C and Small D: FLT3-ITD knockin impairs hematopoietic stem cell quiescence/homeostasis, leading to myeloproliferative neoplasm. Cell Stem Cell 11: 346-358, 2012.

44. Natarajan K, Xie Y, Burcu M, Linn DE, Qiu Y and Baer MR: Pim-1 kinase phosphorylates and stabilizes 130 kDa FLT3 and promotes aberrant STAT5 signaling in acute myeloid leukemia with FLT3 internal tandem duplication. PLoS One 8: e74653, 2013.

45. Mohsin S, Khan M, Nguyen J, Alkatib M, Siddiqi S, Hariharan N, Wallach K, Monsanto M, Gude N, Dembitsky W and Sussman MA: Rejuvenation of human cardiac progenitor cells with Pim-1 kinase. Circ Res 113: 1169-1179, 2013.

46. Linn DE, Yang X, Sun F, Alkatib M, Siddiqi S, Hariharan N, Wallach K, Monsanto M, Gude N, Dembitsky W and Sussman MA: A role for OCT4 in tumor initiation of drug-resistant prostate cancer cells. Genes Cancer 1: 908-916, 2010.

47. Zhou S, Morris JJ, Barnes Y,Lan L, Schuetz JD and Sorrentino BP: Bcrp1 gene expression is required for normal numbers of side population stem cells in mice, and confers relative protection to mitoxantrone in hematopoietic cells in vivo. Proc Natl Acad Sci USA 99: 12339-12344, 2002.

48. Foster BA, Gangavarapu KJ, Mathew G, Azabdaftari G, Morrison CD, Miller A and Huss WJ: Human prostate side population cells demonstrate stem cell properties in recombination with urogenital sinus mesenchyme. PLoS One 8: e55062, 2013.

49. Patrawala L, Calhoun T, Schneider-Broussard R, Zhou J, Claypool K and Tang DG: Side population is enriched in tumorigenic, stem-like cancer cells, whereas ABCG2+ and ABCG2- cancer cells are similarly tumorigenic. Cancer Res 65: 6207-6219, 2005

50. Linn DE: The role of Pim-1 kinases in advanced prostate cancer therapeutic resistance. Ph.D. dissertation. University of Maryland School of Medicine.

51. Brumbaugh J, Hou Z, Russell JD, Howden SE, Yu P, Ledvina AR, Coon JJ and Thomson JA: Phosphorylation regulates human OCT4. Proc Natl Acad Sci USA 109: 7162-7168, 2012

52. Li Y, Li A, Glas M, Lal B, Ying M, Sang Y, Xia S, Trageser D, Guerrero-Cázares H, Eberhart CG, et al: c-Met signaling induces a reprogramming network and supports the glioblastoma stem-like phenotype. Proc Natl Acad Sci USA 108: 9951-9956, 2011.

53. van Leenders GJ, Sookhlall R, Teubel WJ, de Ridder CM, Reneman S, Sacchetti A, Vissers KJ, van Weerden W and Jenster G: Activation of c-MET induces a stem-like phenotype in human prostate cancer. PLoS One 6: e26753, 2011.

54. Cen B, Xiong Y, Song JH, Mahajan S, DuPont R, McEachern K, DeAngelo DJ, Cortes JE, Minden MD, Ebens A, et al: The Pim-1 protein kinase is an important regulator of MET receptor tyrosine kinase levels and signaling. Mol Cell Biol 34: 2517-2532, 2014.

55. Xie Y, Lu W, Liu S, Yang Q, Carver BS, Li E, Wang Y, Fazli L, Gleave $\mathrm{M}$ and Chen Z: Crosstalk between nuclear MET and SOX $/ / \beta$-catenin correlates with castration-resistant prostate cancer. Mol Endocrinol 28: 1629-39, 2014.

56. Mumenthaler SM, Ng PY, Hodge A, Bearss D, Berk G, Kanekal S, Redkar S, Taverna P, Agus DB and Jain A: Pharmacologic inhibition of Pim kinases alters prostate cancer cell growth and resensitizes chemoresistant cells to taxanes. Mol Cancer Ther 8: 2882-2893, 2009.

57. Kirschner AN, Wang J, van der Meer R, Anderson PD, Franco-Coronel OE, Kushner MH, Everett JH, Hameed O, Keeton EK, Ahdesmaki M, et al: PIM kinase inhibitor AZD1208 for treatment of MYC-driven prostate cancer. J Natl Cancer Inst 107: dju407, 2014. 